\title{
Dimensional Effects on Magnetic Properties of Fe-Si Steels due to Laser and Mechanical Cutting
}

\author{
E. Gomes*, J. Schneider*, K. Verbeken*, G. Pasquarella ${ }^{\dagger}$ and Y. Houbaert* \\ ${ }^{*}$ Ghent University, Department of Materials Science and Engineering, Technologiepark 903, B-9052 Ghent, Belgium \\ ${ }^{\dagger}$ LCD LaserCut AG, CH-5026 Densbüren, Switzerland
}

\begin{abstract}
Microstructural deterioration near the cut line and presence of residual stresses both affect the magnetic properties of cut parts. In this paper, the differences between microstructural deterioration resulting from mechanical and laser cutting as well as the sample size effects observed upon hysteresis shall be discussed. It shall be shown that the underlying mechanism for changes in magnetic properties due to mechanical cutting is distinct from that of laser cutting.
\end{abstract}

Index Terms-Cutting process, Magnetic anisotropy, Magnetic deterioration, Silicon steel

\section{INTRODUCTION}

It is well established that each cutting technique affects the properties of the cut zone differently [1]-[8]. When it comes to mechanical cutting, plastic deformation becomes clearly visible near the cut line, whereas laser cutting induces a thermal shock wave, which results in thermal stresses. As a consequence, the material's magnetic properties near the cut are influenced. Microstructural changes at the cut edge have been intensively investigated through micro-hardness measurements, examination of grain morphology changes (with for instance optical microscopy) and evaluation of crystallographic texture variations as well as of misorientation gradients by using Electron Backscatter Diffraction (EBSD) [1], [2], [4]. The variation of the magnetic flux distribution near the cut edge was also studied [3], [9]. Micro-hardness [4] and flux density variation [3] measurements near the cut edge indicate much less deterioration of magnetic properties after laser cutting when compared to mechanical cutting, although this is reported not always to be the case. Less attention has been given to the deterioration of magnetic properties such as permeability and specific magnetic losses as a function of sample size after applying different cutting techniques.

\section{EXPERIMENTAL PROCEDURE}

The investigated samples comprise commercially produced non-oriented $\mathrm{Fe}-\mathrm{Si}$ steels with thickness of $0.5 \mathrm{~mm}$ and medium silicon content.,as well as FeSi6.5 steel. Strips with thickness of $0.2 \mathrm{~mm}$ and with different width $(5 \mathrm{~mm}$ to $30 \mathrm{~mm})$ as well as rings with different inner radius $R_{i}$ and fixed outer radius $R_{a}$, i.e. $\left(R_{a}-R_{i}=15,10\right.$ and $\left.5 \mathrm{~mm}\right)$, were prepared through mechanical and laser cutting. The microstructure was studied by optical metallography and EBSD. The microhardness as a function of the distance from the cut edge was measured using a Zwick ${ }^{\circledR}$; machine with a load of $0.2 \mathrm{~kg}$. The accuracy of every measurement is smaller than the values defined by

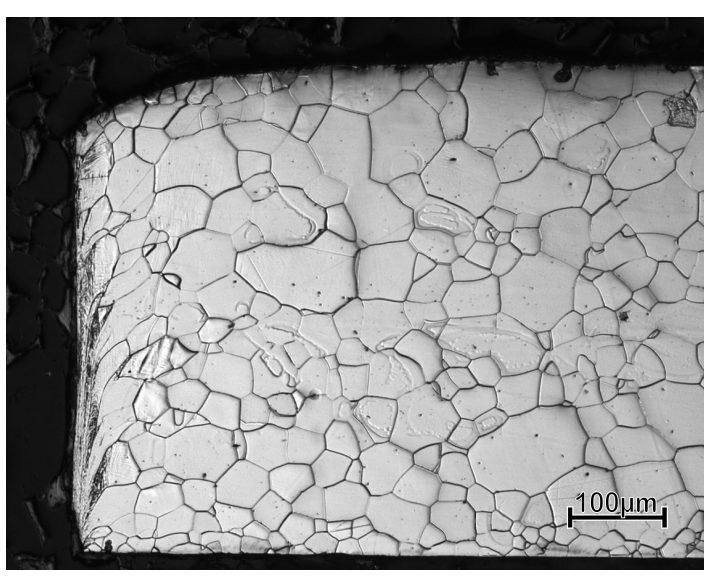

Figure 1. Optical micrograph of a Fe-Si steel with medium Si-content after mechanical cutting.

the ISO/DIS 6507-2:2005 standard. The hysteresis loops were observed using a Brockhaus ${ }^{\circledR}$; magnetic measurement unit. The magnetic measurements were performed at $50 \mathrm{~Hz}$. The magnetic field was applied in the rolling direction.

\section{RESULTS}

\section{A. Microstructure}

Fig. 1 shows the grain morphology of a Fe-Si steel with medium Si-content after mechanical cutting. The grain morphology is as observed elsewhere [4], [10]. As described in literature [1], [2], [4] plastic deformation near the cut line (left side of the figure) can be clearly seen. As is shown in Fig. 2, laser cutting does not induce any changes in the grain morphology nearby the cut line. This was also reported in literature [1], [2], [4].

Fig. 3 demonstrates the texture evolution starting from an area near the cut edge for a sample prepared by laser cutting by making use of the $\varphi_{2}=45^{\circ}$ section of an Orientation Distribution Function (ODF) obtained by EBSD. The same sample as shown in Fig. 2 was measured and ODFs were calculated starting at the cut edge including all grains for a distance of $50 \mu \mathrm{m}$ (region A) and $140 \mu \mathrm{m}$ (region B) as well as inside the material. Although the statistics are rather poor, because of the small number of grains in the area affected by laser cutting, it was observed in this work that in the A and $\mathrm{B}$ area orientations appeared that were away from the gamma fibre $(\{111\}<u v w>)$. The intensity lines on the ODFs 


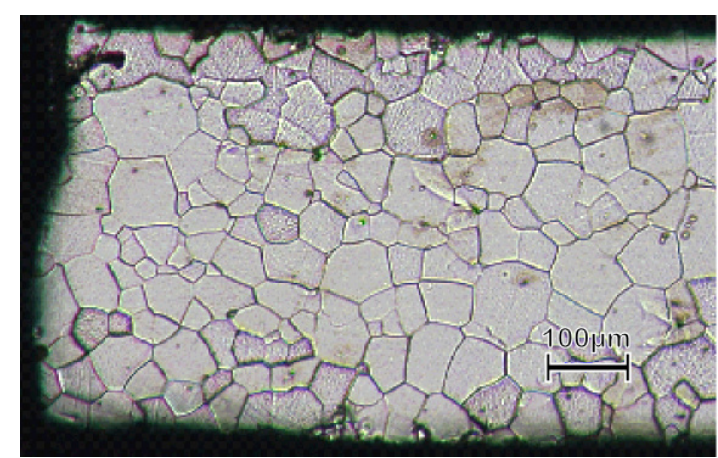

Figure 2. Optical micrograph of a Fe-Si steel with medium Si-content after laser cutting.

of region A are mainly concentrated along the $\{\mathrm{h} 11\}<1 / \mathrm{h}, 1,2>$ fibre. The extension of the zone with different orientations compared to the orientations inside the material is smaller than $200 \mu \mathrm{m}$. Mechanical cutting gives rise to many changes in the crystallographic orientations near the cut line. Due to the heavy cold deformation, it appeared to be very difficult to obtain an acceptable quality of indexation of the diffraction patterns during EBSD measurements. This was reported in similar work by M'Saoubi and Ryde [8]. Therefore, no details could be retrieved from these measurements on local orientation changes and gradients.

\section{B. Microhardness}

Fig. $4 \mathrm{a}$ and $\mathrm{b}$ show the microhardness (Vickers) as a function of the distance from the cut line for the same Fe$\mathrm{Si}$ steel after mechanical and laser cutting. The measurements were repeated three times along two lines perpendicular to the cut edge. The data given in Fig. 4 are average values. While there is an increase of the microhardness in the area near the cut line for mechanical cutting, the microhardness is more or less constant after laser cutting. Similar observations have been realized for quite different steels in [2], [4], [8]. We observed that the zone with increased values of the microhardness after mechanical cutting may go up to $1000 \mu \mathrm{m}$ or even higher [4]. This observation can be correlated with the fact that the cold deformation remains present after mechanical cutting, while laser cutting is a high temperature process that does not cause a higher hardness in the material.

\section{Magnetic Measurements}

Fig. 5 represents the hysteresis loops for the laser cut rings of non-oriented $\mathrm{Fe}-\mathrm{Si}$ steel with medium silicon content and with different inner radius $R_{i}$ and fixed outer radius $R_{a}$, i.e. $\left(R_{a}-R_{i}=15,10\right.$ and $\left.5 \mathrm{~mm}\right)$ and a laser cut Epstein strip with a width of $30 \mathrm{~mm}$ of the same material. Fig. 6 shows the hysteresis loops for strips of FeSi6.5 that were also laser cut with a width of $30,15,10$, and $5 \mathrm{~mm}$. In addition, Fig. 7 gives the hysteresis loops for a strip and a ring of FeSi6.5 both laser cut and with a width of $5 \mathrm{~mm}$. All loops have been measured in maximum fields up to $2000 \mathrm{~A} / \mathrm{m}$ as well as $5000 \mathrm{~A} / \mathrm{m}$. The observed hysteresis loops indicate
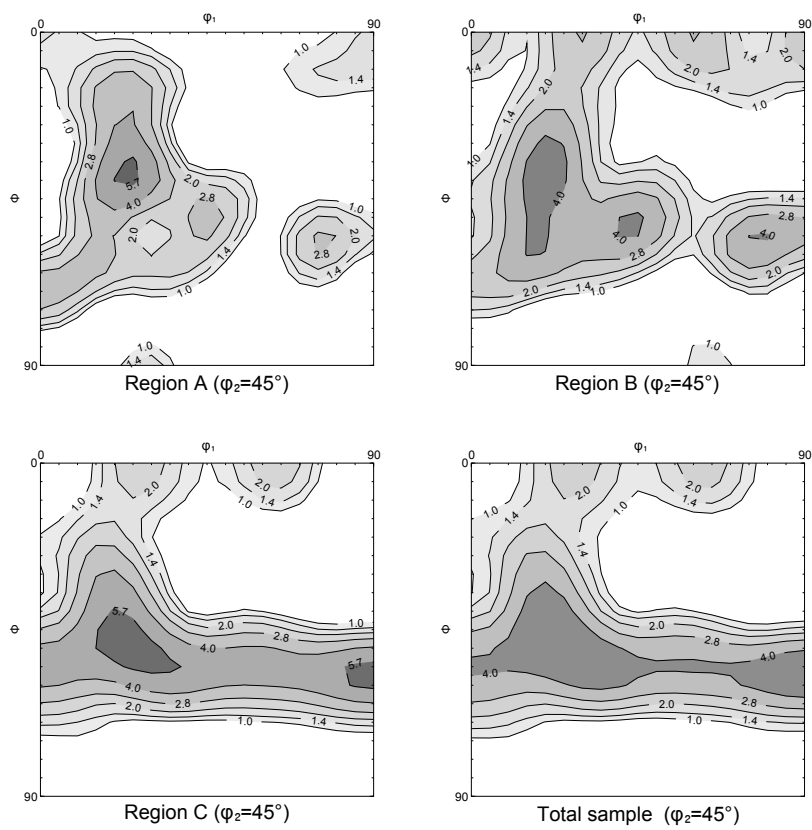

Figure 3. $\varphi_{2}=45^{\circ}$ section of ODF obtained by EBSD of a Fe-Si steel with medium Si-content after laser cutting as a function of the distance from the cutting edge: A - $50 \mu \mathrm{m} ; \mathrm{B}-140 \mu \mathrm{m} ; \mathrm{C}$ - inside the material. Levels: 1.0 $1.4-2.0-2.8-4.0-5.7$.
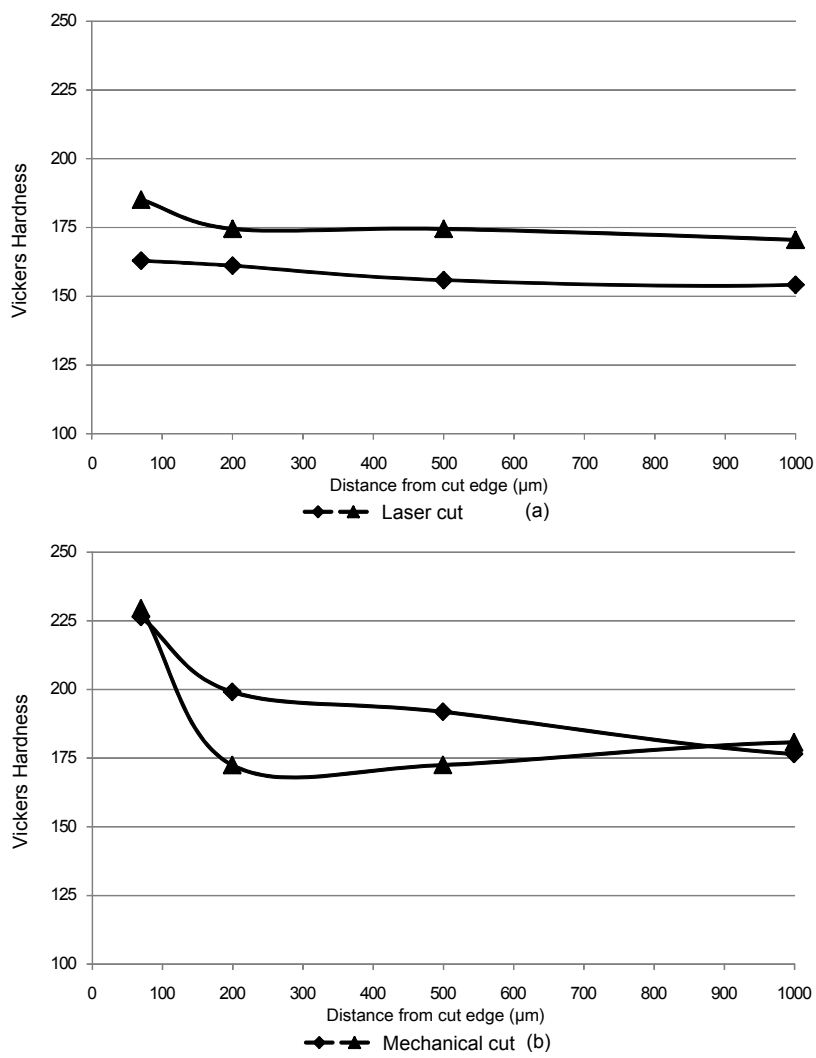

Figure 4. Microhardness of a Fe-Si steel with medium Si-content after laser cutting (a) and mechanical cutting (b). 


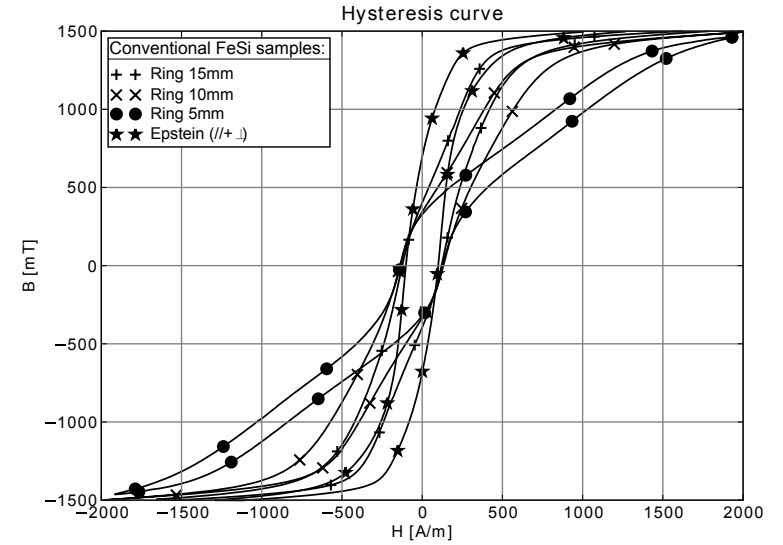

Figure 5. Hysteresis loops for laser cut rings of conventional $\mathrm{Fe}-\mathrm{Si}$ steel with different inner radius $R_{i}$ and fixed outer radius $R_{a} .\left(R_{a}-R_{i}=15,10\right.$, and $5 \mathrm{~mm}$ ); applied field strength up to $2000 \mathrm{~A} / \mathrm{m}$.

clearly a "dimensional effect". There appears qualitatively no difference between ring and strip samples as shown in Fig. 7. The magnetizing behavior becomes increasingly worse, and the permeability decreases, at decreasing width compared to the Epstein strip in the induction range of $0.5 \mathrm{~T}$ to $1.5 \mathrm{~T}$. The decrease of the permeability in the induction range of $0.5 \mathrm{~T}$ to 1.5T is much smaller for samples of FeSi6.5 prepared by laser cutting. The coercive field strength when the maximum field goes up to $2000 \mathrm{~A} / \mathrm{m}$ is practically the same for all ring samples despite the quite different widths as can be seen from Fig. 6 . The same observation holds for the strips of conventional Fe$\mathrm{Si}$ and of FeSi6.5 (Fig. 6 and Fig. 7). However, the value of $B_{r}$ decreases with decreasing width value in both cases: i.e. for rings and strips. The lower values of $B_{r}$ lead to lower values of the permeability, as observed. This may be attributed to the appearance of an additional magnetic anisotropy with a preferred axis perpendicular to the applied field direction. This may originate from a semi macroscopic residual stress in the samples, i.e. an internal stress distributed over several grains) obtained by laser cutting. During laser treatment biaxial stresses: tensile as well as compressive stresses appear as was demonstrated elsewhere [11], [12]. The resulting stressinduced magnetic anisotropy is proportional to the magnitude of this residual stress and the value of the magnetostriction. The lower values of the magnetostriction for FeSi6.5 compared to the conventional $\mathrm{Fe}-\mathrm{Si}$ steel materials may explain why the effect is much larger for the conventional $\mathrm{Fe}-\mathrm{Si}$ steels. This explanation is supported by the fact that we observed even larger effects for soft magnetic Fe-Co samples, which exhibit a larger value of magnetostriction compared to the $\mathrm{Fe}-\mathrm{Si}$ steels [13].

\section{DISCUSSION}

The observed changes microstructural and the appearance of residual stresses affect the magnetizing behavior in quite a different way. Local changes of grain size and texture result in local changes of the critical field for domain wall movement.

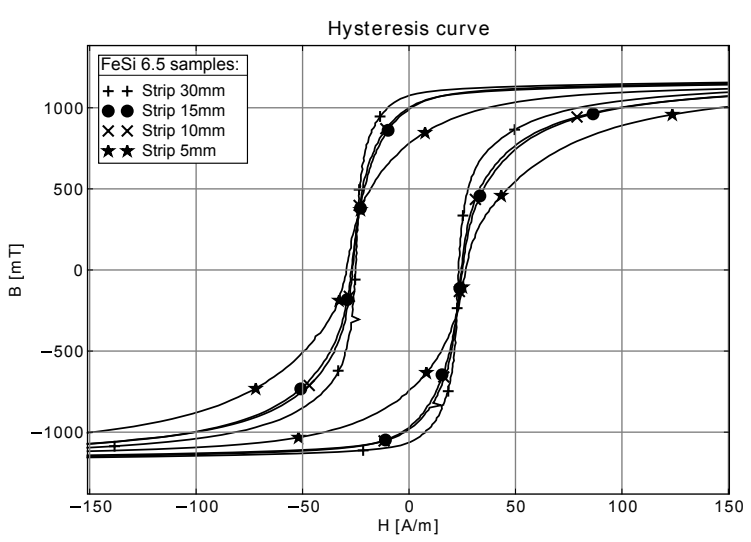

Figure 6. Hysteresis loops for laser cut strips of FeSi6.5 with a width of 30, 15,10 and $5 \mathrm{~mm}$; applied field strength up to $2000 \mathrm{~A} / \mathrm{m}$.

Stress induced magnetic anisotropy gives rise to changes of the remanent induction and the permeability at higher applied magnetic fields. In previous work [7], [10] we studied the influence of grain size for low and medium non oriented $\mathrm{Fe}-$ $\mathrm{Si}$ materials on the deterioration of the magnetic properties at mechanical cutting. We found that the magnetizing behavior became generally worse at large grain size as well as smaller sample width (dimensional effect). Fig. 8 shows the behaviour of the exciting field to reach $1.5 \mathrm{~T}$ for the different samples with variable grain size and width. The trend is similar for mechanical cutting using sharp or less sharp cutting tool. Comparing the obtained results for mechanical and laser cutting a quite different behavior is observed. While for the same Fe-Si material grade as in Fig. 5 after mechanical cutting and magnetizing the sample to $1.0 \mathrm{~T}$ at $50 \mathrm{~Hz}$ a decrease of the induction B in the area at the cut line was observed in [3], no such decrease appeared for laser cutting. The area, where a decrease of the induction $\mathrm{B}$ is observed after mechanical cutting correlates with the area of enhanced microhardness, which may originate from the elastic and plastic deformation induced by mechanical cutting. The observed decrease in permeability with decreasing value of the width of the strips, and the increase of the magnetizing field, in the range of $0.5 \mathrm{~T}$ up to $1.5 \mathrm{~T}$ after mechanical cutting of conventional $\mathrm{Fe}-\mathrm{Si}$ steels [6], [7] show a clear dependence of the mean grain size of the material as shown in Fig. 8 [10].

Plastic deformation can be clearly seen after mechanical cutting, while the grain morphology of the samples prepared by laser cutting, as shown in Fig. 2, is the same in the area near the cut line as inside the material (far away from the cut line). The observed decrease of the permeability, and the increase of the magnetizing field to reach a certain value of B using laser cutting is quite different compared to mechanical cutting for samples with nearly the same grain size, see Fig. 8. The decrease of the permeability, respectively the increase of the magnetizing field, with decreasing width value for strips in the range of $0.5 \mathrm{~T}$ up to $1.5 \mathrm{~T}$ becomes larger with increasing grain size at mechanical cutting, while it becomes 


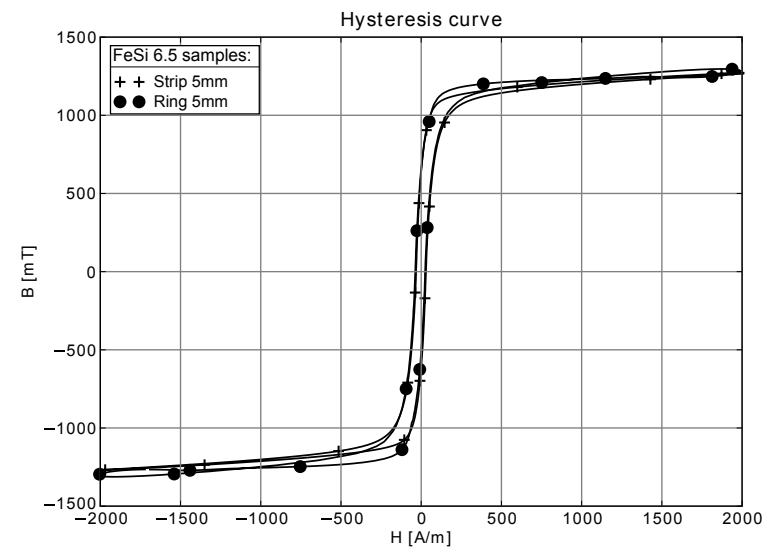

Figure 7. Hysteresis loops for a laser cut ring and strip of FeSi6.5 with a width of $5 \mathrm{~mm}$; applied field strength up to $2000 \mathrm{~A} / \mathrm{m}$.

smaller at increasing grain size after laser cutting. These facts point to different underlying deterioration mechanisms of the magnetic properties for the two cutting techniques. In the case of mechanical cutting there is a clear region of changes in the grain morphology near the cut edge due to elastic and plastic deformation. On the other hand, there is no clear indication of a change of the grain morphology for samples obtained by laser cutting. Residual biaxial stresses due to the thermal shock wave at laser cutting may be therefore the origin of the observed changes of the remanent induction, respectively the decrease of the permeability at higher magnetic fields. The resulting stress-induced magnetic anisotropy is proportional to the magnitude of the residual biaxial stress and the value of the magnetostriction. The magnetostriction, itself depends on the silicon content and becomes smaller for increasing the $\mathrm{Si}$ content.

\section{CONCLUSiOnS}

Although the appearance of the microstructure after cutting is quite different, both mechanical and laser cutting cause a worsening of the magnetic properties of the electrical steels. Additional deterioration originates from the dimension of the samples, which will also have to reflect itself in the optimum choice of the material grade to reach a minimal deterioration.

\section{ACKNOWLEDGMENT}

The authors would like to thank N. Sanchez for the help with the EBSD measurements. Kim Verbeken is a Postdoctoral Fellow with the Fund for Scientific Research - Flanders (Belgium) (F.W.O.-Vlaanderen).

\section{REFERENCES}

[1] M. Emura, F. Landgraf, W. Ross, and J. Barreta, "The influence of cutting technique on the magnetic properties of electrical steels," Journal of Magnetism and Magnetic Materials, vol. 254, pp. 358-360, 2003.

[2] Y. Kurosaki, H. Mogi, H. Fujii, T. Kubota, and M. Shiozaki, "Importance of punching and workability in non-oriented electrical steel sheets," Journal of Magnetism and Magnetic Materials, vol. 320, pp. 2474-2480, 2008.

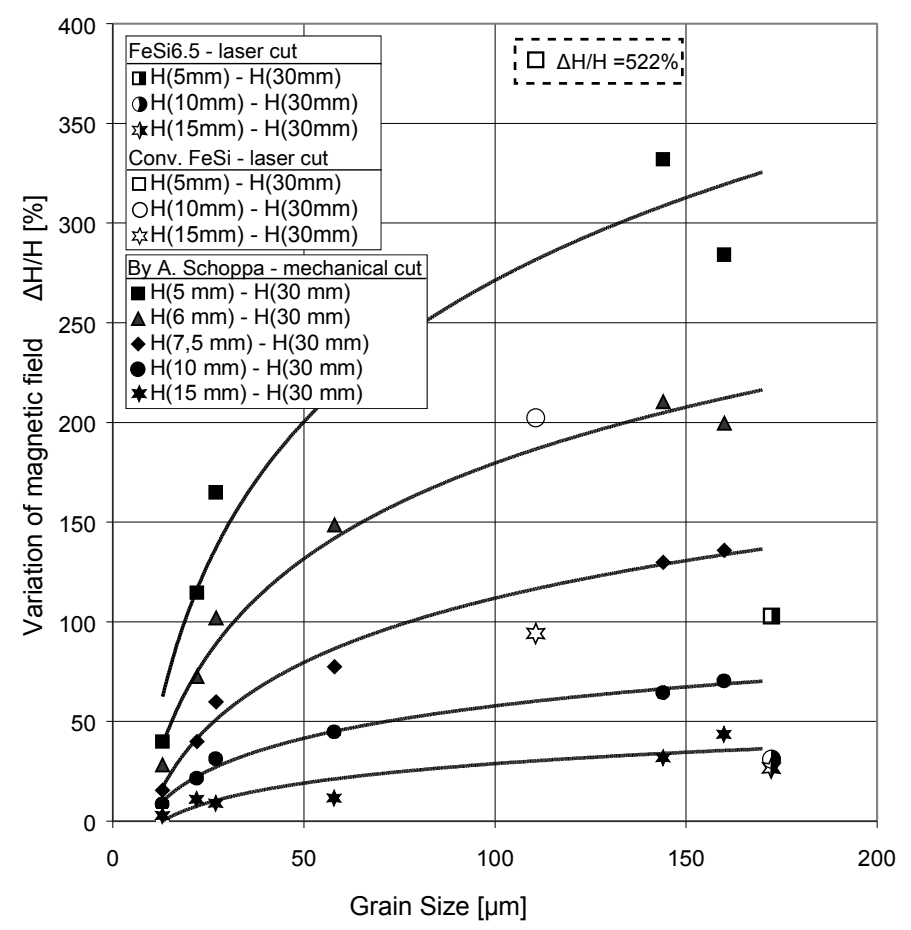

Figure 8. Increase of the magnetizing field $\Delta H$ to reach $\mathrm{B}=1 \mathrm{~T}$ as a function of the grain size at mechanical cutting [10] and data for laser cut samples; $\Delta H=H_{x}-H_{30 m m}$.

[3] G. Loisos and A. J. Moses, "Effect of mechanical and Nd:YAG laser cutting on magnetic flux distribution near the cut edge of non-oriented steels," Journal of Materials Processing Technology, vol. 161, pp. 151155, 2005.

[4] G. Pasquarella and J. Schneider, "Electric machine innovation by integrated laser cutting technology," in Proceedings Conference WMM Gent, 2008.

[5] K. H. Schmidt, "Influence of punching on the magnetic properties of electric steel with 1\% silicon," Journal of Magnetism and Magnetic Materials, vol. vol.2, no.1-3, pp. 136-50, 1975.

[6] A. Schoppa, J. Schneider, and J. O. Roth, "Influence of the cutting process on the magnetic properties of non-oriented electrical steels," Journal of Magnetism and Magnetic Materials, vol. 215, pp. 100-102, 2000.

[7] A. Schoppa, J. Schneider, and C. D. Wuppermann, "Influence of the manufacturing process on the magnetic properties of non-oriented electrical steels," Journal of Magnetism and Magnetic Materials, vol. 215, pp. 74-78, 2000.

[8] R. M'Saoubi and L. Ryde, "Application of the EBSD technique for the characterisation of deformation zones in metal cutting," Materials Science \& Engineering A, vol. 405, pp. 339-349, 2005.

[9] R. Rygal, A. J. Moses, N. Derebasi, J. Schneider, and A. Schoppa, "Influence of cutting stress on magnetic field and flux density distribution in non-oriented electrical steels," Journal of Magnetism and Magnetic Materials, vol. 215, pp. 687-689, 2000.

[10] A. Schoppa, "Einfluß der Be- und Verarbeitung auf die Eigenschaften von schlußgeglühtem, nichtkornorientierten Elektroband," Ph.D. dissertation, RWTH Aachen, Germany, 2001.

[11] G. Lu, A. Kotousov, and E. Siores, "Elementary mathematical theory of thermal stresses and fracture during welding and cutting," Journal of Materials Processing Technology(Netherlands), vol. 89, pp. 298-302, 1999.

[12] M. Zain-ul Abdein, D. Nelias, J. Jullien, and D. Deloison, "Prediction of laser beam welding-induced distortions and residual stresses by numerical simulation for aeronautic application," Journal of Materials Processing Tech., vol. 209, no. 6, pp. 2907-2917, 2009.

[13] E. Gomes and J. Schneider, "To be published." 